Research Article

\title{
Yosida Complementarity Problem with Yosida Variational Inequality Problem and Yosida Proximal Operator Equation Involving XOR-Operation
}

\author{
Rais Ahmad $\mathbb{D}^{1},{ }^{1}$ Arvind Kumar Rajpoot $\mathbb{D}^{,},{ }^{1}$ Imran Ali $\mathbb{D}^{2},{ }^{2}$ and Ching-Feng Wen $\mathbb{D}^{3,4}$ \\ ${ }^{1}$ Department of Mathematics, Aligarh Muslim University, Aligarh 202002, India \\ ${ }^{2}$ Department of Mathematics, School of Advanced Sciences, Kalasalingam Academy of Research and Education, Anand Nagar, \\ Krishnankoil 626126, India \\ ${ }^{3}$ Center for Fundamental Science, Research Center for Nonlinear Analysis and Optimization, Kaohsiung Medical University, \\ Kaohsiung 80708, Taiwan \\ ${ }^{4}$ Department of Medical Research, Kaohsiung Medical University Hospital, Kaohsiung 80708, Taiwan
}

Correspondence should be addressed to Ching-Feng Wen; cfwen@kmu.edu.tw

Received 3 September 2021; Revised 7 October 2021; Accepted 1 November 2021; Published 27 November 2021

Academic Editor: Antonio Di Crescenzo

Copyright $\odot 2021$ Rais Ahmad et al. This is an open access article distributed under the Creative Commons Attribution License, which permits unrestricted use, distribution, and reproduction in any medium, provided the original work is properly cited.

Due to the importance of Yosida approximation operator, we generalized the variational inequality problem and its equivalent problems by using Yosida approximation operator. The aim of this work is to introduce and study a Yosida complementarity problem, a Yosida variational inequality problem, and a Yosida proximal operator equation involving XOR-operation. We prove an existence result together with convergence analysis for Yosida proximal operator equation involving XOR-operation. For this purpose, we establish an algorithm based on fixed point formulation. Our approach is based on a proximal operator technique involving a subdifferential operator. As an application of our main result, we provide a numerical example using the MATLAB program R2018a. Comparing different iterations, a computational table is assembled and some graphs are plotted to show the convergence of iterative sequences for different initial values.

\section{Introduction}

Stampacchia [1] and Ficchera [2] originated the study of variational inequalities, separately. Variational inequalities are mathematical models for many problems occurring in physics, engineering sciences, transportation planning, financial problems, and in many industrial strategies, etc. (see, for example, [3-11]). In 1968, Cottle and Dantzig [12] proposed linear complementarity problem which appear continually in computational mechanics. It is interesting to note that finding the solution of linear complementarity problem is associated with minimizing some quadratic function. However, in 1964, Cottle [13] in his Ph. D thesis introduced nonlinear complementarity problem which is closely related to Hartman and Stampacchia variational inequality problem. The proximal operator technique is useful to establish equivalence between variational inequalities and proximal operator equations. The proximal operator equation approach is used to solve variational inequalities and related optimization problems.

$\mathrm{XOR}$ is a logical operation and represents the inequality function, that is, the output is true if the inputs are not alike; otherwise, the output is false. An easy way to remember XOR is "must have one or the other but not both." It is important to note that XOR does not leak information about the original plain text. The inner XOR is the encryption and the outer XOR is the decryption, that is, the exact XOR function can be used for both encryption and decryption. Consider a string of binary digits 10101 and XOR the string 10111 with it to get 00010 . That is, the original string is encoded and the second string becomes key; if we XOR our key with our encoded string, we get our original string back. XOR allows 
to easily encrypt and decrypt a string; the other logical operations do not.

The possible strategy of solving stochastic notion of multivalued differential equation in finite dimensional space is based on Yosida approximation approach. The existence of multivalued stochastic differential equation in finite dimensional space with a time-independent, deterministic maximal monotone operator through Yosida approximation approach was first discussed by Petterson [14]. Yosida approximation operators are used to solve wave equations, heat equations, etc. For more details and recent past developments about complementarity problems, variational inequalities, proximal operator equations, Yosida approximation operator, and related topics, we refer to [15-28] and references therein.

Motivated by all the above discussed concepts, in this paper, we consider and study a Yosida complementarity problem, a Yosida variational inequality problem, and a Yosida proximal operator equation involving XOR-operation. Some equivalence results are proved. To obtain the solution of Yosida proximal operator equation involving XOR-operation, we define an algorithm based on fixed point formulation. Convergence criteria are also discussed. In support of our main result, an example is provided using MATLAB program R2018a. A comparison of different iterations is assembled in the form of a computational table, and the convergence of the iterative sequences is shown by some graphs for different initial values.

\section{Preliminaries and Basic Results}

We suppose that $H$ is a real ordered positive Hilbert Space with its norm $\|\cdot\|$ and inner product $\langle\cdot, \cdot\rangle, \mathscr{C} \subseteq H$ is a closed convex pointed cone, $d$ is the metric induced by the norm $\|\cdot\|, \widetilde{C} B(H)$ is the family of nonempty, closed, and bounded subsets of $H$, and $D(\cdot, \cdot)$ is the Hausdorff metric on $\widetilde{C} B(H)$.

The following definitions, concepts, and results are required for the presentation of this paper.

$$
\left\|N\left(u_{1}, \cdot, \cdot\right)-N\left(u_{2}, \cdot, \cdot\right)\right\| \leq \lambda_{N_{1}}\left\|u_{1}-u_{2}\right\|
$$

$\forall x_{1}, x_{2} \in \mathscr{C}, u_{1} \in A\left(x_{1}\right)$ and $u_{2} \in A\left(x_{2}\right)$

(ii) $N$ is said to be Lipschitz continuous in the second argument if there exists a constant $\lambda_{N_{2}}>0$ such that

Definition 1. A convex cone is a subset of a vector space over an ordered field that is closed under linear combinations with positive coefficients.

Definition 2. Two elements $x$ and $y$ of a set $X$ are said to be comparable with respect to a binary operation $\leq$, if at least one of $x \leq y$ or $y \leq x$ is true. Comparable elements $x$ and $y$ are denoted by $x \propto y$.

Definition 3. A partial order is any binary relation which is reflexive, antisymmetric, and transitive.

Definition 4. Suppose $\operatorname{lub}\{x, y\}$ and $\operatorname{glb}\{x, y\}$ for the set $\{x, y\}$ exist; then, XOR and XNOR operations denoted by $\oplus$ and $\odot$ are defined as follows:

(i) $x \oplus y=(x-y) \vee(y-x)$

(ii) $x \odot y=(x-y) \wedge(y-x)$, where $x \vee y=\operatorname{lub}\{x, y\}$, $x \wedge y=\operatorname{glb}\{x, y\}$, lub means the least upper bound, and glb means the greatest lower bound

Proposition 1 (see [29]). Let $\oplus$ be an XOR-operation and $\odot$ be an XNOR operation. Then, the following axioms are true:
(i) $x \odot x=0, x \odot y=y \odot x=-(x \oplus y)=-(y \oplus x)$
(ii) $x \oplus x=0, x \oplus y=y \oplus x, 0 \leq x \oplus y$
(iii) $x \oplus 0=x$
(iv) $0 \leq x \oplus y$, if $x \propto y$
(v) If $x \propto y$, then $x \oplus y=0$ if and only if $x=y$
(vi) $\|0 \oplus 0\|=\|0\|$
(vii) $\|x \oplus y\| \leq\|x-y\|$
(viii) If $x \propto y$, then $\|x \oplus y\|=\|x-y\|$

Definition 5. Let $N: H \times H \times H \longrightarrow H$ be a single-valued mapping and $A: \mathscr{C} \longrightarrow \widetilde{C} B(H)$ be a multivalued mapping. Then

(i) $N$ is said to be Lipschitz continuous in the first argument if there exists a constant $\lambda_{N_{1}}>0$ such that

$$
\left\|N\left(\cdot, u_{1}, \cdot\right)-N\left(\cdot, u_{2}, \cdot\right)\right\| \leq \lambda_{N_{2}}\left\|u_{1}-u_{2}\right\|, \quad \forall x_{1}, x_{2} \in \mathscr{C}, u_{1} \in A\left(x_{1}\right) \text { and } u_{2} \in A\left(x_{2}\right)
$$

Similarly, we can define the Lipschitz continuity of $N$ in the third argument. 
Definition 6. A multivalued mapping $A: \mathscr{C} \longrightarrow \widetilde{C} B(H)$ is said to be $D$-Lipschitz continuous if for any $x, y \in \mathscr{C}$, there exists a constant $\lambda_{D_{A}}>0$ such that

$$
D(A(x), A(y)) \leq \lambda_{D_{A}}\|x-y\| .
$$

Definition 7 (see [30]). Let $\psi: H \longrightarrow \mathbb{R} \cup\{+\infty\}$ be a proper convex functional. A vector $w \in H$ is called subgradient of $\psi$ at $x \in d o m \psi$, if

$$
\langle w, y-x\rangle \leq \psi(y)-\psi(x), \quad \forall y \in H .
$$

The set of all subgradients of $\psi$ at $x$ is denoted by $\partial \psi(x)$. The mapping $\partial \psi: H \longrightarrow 2^{H}$ defined by

$$
\partial \psi(x)=\{w \in H:\langle w, y-x\rangle \leq \psi(y)-\psi(x), \forall y \in H\}
$$

is called subdifferential of $\psi$.

Definition 8. Let $P: \mathscr{C} \longrightarrow \mathscr{C}$ be a mapping and $\psi: \mathscr{C} \longrightarrow \mathbb{R} \cup\{+\infty\}$ be a proper convex functional. The proximal operator $J_{\rho}^{\partial \psi}: \mathscr{C} \longrightarrow \mathscr{C}$ is defined by

$$
J_{\rho}^{\partial \psi}(x)=[P+\rho \partial \psi]^{-1}(x), \quad \forall x \in \mathscr{C},
$$

where $\rho>0$ is a constant.

Definition 9. The Yosida approximation operator of $\psi$ is defined by

$$
Y_{\rho}^{\partial \psi}(x)=\frac{1}{\rho}\left[I-J_{\rho}^{\partial \psi}\right](x), \quad \forall x \in \mathscr{C},
$$

where $\rho>0$ is a constant.

Furthermore, we prove some propositions related to proximal operator and Yosida approximation operator.

Proposition 2. Let $P: \mathscr{C} \longrightarrow \mathscr{C}$ and $\psi: \mathscr{C} \longrightarrow \mathbb{R} \cup\{+\infty\}$ be linear mappings, then the proximal operator $J_{\rho}^{\partial \psi}$ is linear. That is

$$
\alpha J_{\rho}^{\partial \psi}(x)=J_{\rho}^{\partial \psi}(\alpha x)
$$

provided

$$
\begin{array}{r}
\left(J_{\rho}^{\partial \psi}\right)^{-1}\left(J_{\rho}^{\partial \psi}(x)\right)=\left(\left(J_{\rho}^{\partial \psi}\right)^{-1}\left(J_{\rho}^{\partial \psi}\right)\right)(x)=x, \\
\forall x \in \mathscr{C} \text { and } \alpha>0 .
\end{array}
$$

Proof. Using the definition of $J_{\rho}^{\partial \psi}$, linearity of $P$ and $\psi$, and Theorem 1.48 and Theorem 1.49 of [31], we have

$$
\begin{aligned}
\alpha J_{\rho}^{\partial \psi}(x) & =J_{\rho}^{\partial \psi}\left(J_{\rho}^{\partial \psi}\right)^{-1}\left(\alpha J_{\rho}^{\partial \psi}(x)\right) \\
& =J_{\rho}^{\partial \psi}[P+\rho \partial \psi]\left(\alpha J_{\rho}^{\partial \psi}(x)\right) \\
& =J_{\rho}^{\partial \psi}\left[P\left(\alpha J_{\rho}^{\partial \psi}(x)\right)+\rho \partial \psi\left(\alpha J_{\rho}^{\partial \psi}(x)\right)\right] \\
& =J_{\rho}^{\partial \psi}\left[P\left(\alpha J_{\rho}^{\partial \psi}(x)\right)+\rho \partial \psi\left(\alpha J_{\rho}^{\partial \psi}(x)\right)\right] \\
& =J_{\rho}^{\partial \psi}\left[P\left(\alpha J_{\rho}^{\partial \psi}(x)\right)+\rho \partial\left(\alpha \psi\left(J_{\rho}^{\partial \psi}(x)\right)\right)\right] \\
& =J_{\rho}^{\partial \psi}\left[\alpha P\left(J_{\rho}^{\partial \psi}(x)\right)+\rho \alpha \partial \psi\left(J_{\rho}^{\partial \psi}(x)\right)\right] \\
& =J_{\rho}^{\partial \psi}\left[\alpha[P+\rho \partial \psi]\left(J_{\rho}^{\partial \psi}(x)\right)\right] \\
& =J_{\rho}^{\partial \psi}\left[\alpha\left(J_{\rho}^{\partial \psi}\right)^{-1} J_{\rho}^{\partial \psi}(x)\right] \\
& =J_{\rho}^{\partial \psi}\left[\alpha\left(\left(J_{\rho}^{\partial \psi}\right)^{-1}\left(J_{\rho}^{\partial \psi}\right)\right)(x)\right] \\
& =J_{\rho}^{\partial \psi}[\alpha x] .
\end{aligned}
$$

Proposition 3. The Yosida approximation operator $Y_{\rho}^{\partial \psi}: \mathscr{C} \longrightarrow \mathscr{C}$ is linear, that is,

$$
Y_{\rho}^{\partial \psi}(\alpha x)=\alpha Y_{\rho}^{\partial \psi}(x), \quad \forall x \in \mathscr{C} .
$$

Proof. Using the definition of $Y_{\rho}^{\partial \psi}$ and Proposition 2, we have

$$
\begin{aligned}
Y_{\rho}^{\partial \psi}(\alpha x) & =\frac{1}{\rho}\left[I-J_{\rho}^{\partial \psi}\right](\alpha x) \\
& =\frac{1}{\rho}\left[\alpha x-J_{\rho}^{\partial \psi}(\alpha x)\right] \\
& =\frac{1}{\rho}\left[\alpha x-\alpha J_{\rho}^{\partial \psi}(x)\right] \\
& =\frac{\alpha}{\rho}\left[I-J_{\rho}^{\partial \psi}\right](x) \\
& =\alpha Y_{\rho}^{\partial \psi}(x), \quad \forall x \in \mathscr{C} .
\end{aligned}
$$

Proposition 4. The proximal operator $J_{\rho}^{\partial \psi}: \mathscr{C} \longrightarrow \mathscr{C}$ is Lipschitz continuous, provided $P: \mathscr{C} \longrightarrow \mathscr{C}$ is strongly monotone with respect to $J_{\rho}^{\partial \psi}$ with constant $\mu>0, \psi$ is strongly convex with modulus $\lambda>0$, and $J_{\rho}^{\partial \psi}$ is strongly monotone with constant $\sigma>0$, where $\sigma=2 \lambda$.

Proof. Let $x, y \in \mathscr{C}$, then

$$
\begin{aligned}
& J_{\rho}^{\partial \psi}(x)=[P+\rho \partial \psi]^{-1}(x), \\
& J_{\rho}^{\partial \psi}(y)=[P+\rho \partial \psi]^{-1}(y) .
\end{aligned}
$$


Thus,

$$
\begin{aligned}
& \frac{1}{\rho}\left[x-P\left(J_{\rho}^{\partial \psi}(x)\right)\right] \in \partial \psi\left(J_{\rho}^{\partial \psi}(x)\right) \\
& \frac{1}{\rho}\left[y-P\left(J_{\rho}^{\partial \psi}(y)\right)\right] \in \partial \psi\left(J_{\rho}^{\partial \psi}(y)\right) .
\end{aligned}
$$

$$
\begin{aligned}
\sigma\left\|J_{\rho}^{\partial \psi}(x)-J_{\rho}^{\partial \psi}(y)\right\|^{2} & \leq\left\langle\frac{1}{\rho}\left(x-P\left(J_{\rho}^{\partial \psi}(x)\right)\right)-\frac{1}{\rho}\left(y-P\left(J_{\rho}^{\partial \psi}(y)\right)\right), x-y\right\rangle \\
& =\frac{1}{\rho}\left\langle x-y-\left(P\left(J_{\rho}^{\partial \psi}(x)\right)-P\left(J_{\rho}^{\partial \psi}(y)\right)\right), x-y\right\rangle \\
& =\frac{1}{\rho}\left[\langle x-y, x-y\rangle-\left\langle P\left(J_{\rho}^{\partial \psi}(x)\right)-P\left(J_{\rho}^{\partial \psi}(y)\right), x-y\right\rangle\right] .
\end{aligned}
$$

Since $P$ is strongly monotone with respect to $J_{\rho}^{\partial \psi}$ with constant $\mu>0$, we have

$$
\sigma\left\|J_{\rho}^{\partial \psi}(x)-J_{\rho}^{\partial \psi}(y)\right\|^{2} \leq \frac{1}{\rho}\left[\|x-y\|^{2}-\mu\left\|J_{\rho}^{\partial \psi}(x)-J_{\rho}^{\partial \psi}(y)\right\|^{2}\right]
$$

which implies that

$$
\left\|J_{\rho}^{\partial \psi}(x)-J_{\rho}^{\partial \psi}(y)\right\| \leq \theta\|x-y\|, \quad \text { where } \theta=\frac{1}{\sqrt{\sigma \rho+\mu}} .
$$

As $\psi$ is strongly convex with modulus $\lambda>0$, then the proximal operator $J_{\rho}^{\partial \psi}$ is strongly monotone with constant $\sigma>0$, where $\sigma=2 \lambda$ (see [31]). Therefore,
That is, $J_{\rho}^{\partial \psi}$ is Lipschitz continuous.

Proposition 5. The Yosida approximation operator is strongly monotone if all the conditions of Proposition 4 hold.

Proof. Using the Lipschitz continuity of proximal operator $J_{\rho}^{\partial \psi}$, we have

$$
\begin{aligned}
\left\langle Y_{\rho}^{\partial \psi}(x)-Y_{\rho}^{\partial \psi}(y), x-y\right\rangle & =\left\langle\frac{1}{\rho}\left[I-J_{\rho}^{\partial \psi}\right](x)-\frac{1}{\rho}\left[I-J_{\rho}^{\partial \psi}\right](y), x-y\right\rangle \\
& =\frac{1}{\rho}\left[\langle x-y, x-y\rangle-\left\langle J_{\rho}^{\partial \psi}(x)-J_{\rho}^{\partial \psi}(y), x-y\right\rangle\right] \\
& \geq \frac{1}{\rho}\left[\|x-y\|^{2}-\left\|J_{\rho}^{\partial \psi}(x)-J_{\rho}^{\partial \psi}(y)\right\|\|x-y\|\right] \\
& \geq \frac{1}{\rho}\left[\|x-y\|^{2}-\theta\|x-y\|^{2}\right] \\
& =\left(\frac{1-\theta}{\rho}\right)\|x-y\|^{2} \\
& =\delta_{y}\|x-y\|^{2}, \quad \text { where } \delta_{y}=\left(\frac{1-\theta}{\rho}\right) .
\end{aligned}
$$

\section{Description of the Problems and Equivalence Lemmas}

Let $H$ be a real ordered positive Hilbert space and $\mathscr{C} \subseteq H$ be a closed convex pointed cone. Let $A, B, C: \mathscr{C} \longrightarrow \widetilde{C} B(H)$ be the multivalued mappings and $N: H \times H \times H \longrightarrow H$ be a single-valued mapping. Suppose $\psi: \mathscr{C} \longrightarrow \mathbb{R} \cup\{+\infty\}$ is a proper, convex functional and $Y_{\rho}^{\partial \psi}: \mathscr{C} \longrightarrow \mathscr{C}$ is the Yosida approximation operator. We consider the following Yosida complementarity problem involving XOR-operation. 
Find $x \in \mathscr{C}, u \in A(x), v \in B(x), w \in C(x)$ such that

$$
\begin{aligned}
& \left\langle N(u, v, w), Y_{\rho}^{\partial \psi}(x)\right\rangle \oplus \psi\left(Y_{\rho}^{\partial \psi}(x)\right)=0, \\
& \left\langle N(u, v, w), Y_{\rho}^{\partial \psi}(y)\right\rangle \oplus \psi\left(Y_{\rho}^{\partial \psi}(y)\right) \geq 0, \quad \forall y \in \mathscr{C} .
\end{aligned}
$$

From problem (21), one can easily obtain the complementarity problems studied by Huang et al. [32], Yin and Xu [33], Flores-Bazán and López [34], Isac [35, 36] and Farajzadeh and Harandi [37], etc.

In connection with Yosida complementarity problem involving XOR-operation (21), we mention the following Yosida variational inequality problem involving XORoperation.

Find $x \in \mathscr{C}, u \in A(x), v \in B(x), w \in C(x)$ such that

$$
\left\langle N(u, v, w), Y_{\rho}^{\partial \psi}(y)-Y_{\rho}^{\partial \psi}(x)\right\rangle \oplus\left(\psi\left(Y_{\rho}^{\partial \psi}(y)\right)-\psi\left(Y_{\rho}^{\partial \psi}(x)\right)\right)
$$$$
\geq 0, \quad \forall y \in \mathscr{C} \text {. }
$$

In acquaintance with Yosida variational inequality problem involving XOR-operation (22), we mention the following Yosida proximal operator equation involving XOR-operation.

$$
\begin{aligned}
& \left\langle N(u, v, w), Y_{\rho}^{\partial \psi}(x)\right\rangle \oplus \psi\left(Y_{\rho}^{\partial \psi}(x)\right)=0, \\
& \left\langle N(u, v, w), Y_{\rho}^{\partial \psi}(y)\right\rangle \oplus \psi\left(Y_{\rho}^{\partial \psi}(y)\right) \geq 0,
\end{aligned}
$$

$\forall x, y \in \mathscr{C}, u \in A(x), v \in B(x), w \in C(x)$.

$$
\left\langle N(u, v, w), Y_{\rho}^{\partial \psi}(y)\right\rangle \geq \psi\left(Y_{\rho}^{\partial \psi}(y)\right) .
$$

Using the properties of inner product, we can write

$$
\begin{aligned}
\left\langle N(u, v, w), Y_{\rho}^{\partial \psi}(y)-Y_{\rho}^{\partial \psi}(x)\right\rangle= & \left\langle N(u, v, w), Y_{\rho}^{\partial \psi}(y)\right\rangle \\
& -\left\langle N(u, v, w), Y_{\rho}^{\partial \psi}(x)\right\rangle .
\end{aligned}
$$

Also, $\left\langle N(u, v, w), Y_{\rho}^{\partial \psi}(y)\right\rangle \oplus \psi\left(Y_{\rho}^{\partial \psi}(y)\right) \geq 0$, we have $\left\langle N(u, v, w), Y_{\rho}^{\partial \psi}(y)\right\rangle \oplus\left(\psi\left(Y_{\rho}^{\partial \psi}(y)\right) \oplus \psi\left(Y_{\rho}^{\partial \psi}(y)\right) \geq \psi\left(Y_{\rho}^{\partial \psi}(y)\right)\right.$.

By (ii) and (iii) of Proposition 1, we have
Find $x, z \in \mathscr{C}, u \in A(x), v \in B(x), w \in C(x)$ such that

$$
N(u, v, w) \oplus \rho^{-1} R_{\rho}^{\partial \psi}(z)=0,
$$

where $\rho>0$ is a constant, $R_{\rho}^{\partial \psi}=\left[I-P\left(J_{\rho}^{\partial \psi}\right)\right], J_{\rho}^{\partial \psi}$ is the proximal operator, $P: \mathscr{C} \longrightarrow \mathscr{C}$ is a mapping, and $=P\left(Y_{\rho}^{\partial \psi}(x)\right)+\rho N(u, v, w)$. and the equivalence between problem (22) and problem (23) are given as follows.

Lemma 1. Let $A, B, C: \mathscr{C} \longrightarrow \widetilde{C} B(H)$ be the multivalued mappings and $\mathrm{N}: \mathrm{H} \times \mathrm{H} \times \mathrm{H} \longrightarrow \mathrm{H}$ be a single-valued mapping. Suppose $\psi: \mathscr{C} \longrightarrow \mathbb{R} \cup\{+\infty\}$ is a linear, proper functional. Let $Y_{\rho}^{\partial \psi}: \mathscr{C} \longrightarrow \mathscr{C}$ be the Yosida approximation $x \in \mathscr{C}, u \in A(x), v \in B(x), w \in C(x)$, then the Yosida complementarity problem involving XOR-operation (21) and the Yosida variational inequality problem involving XOR-operation (22) are equivalent.

Proof. Let the Yosida complementarity problem involving XOR-operation (21) holds. We have
Proposition 1, we have

$$
\left\langle N(u, v, w), Y_{\rho}^{\partial \psi}(x)\right\rangle=\psi\left(Y_{\rho}^{\partial \psi}(x)\right) .
$$

$$
\begin{aligned}
& \left\langle N(u, v, w), Y_{\rho}^{\partial \psi}(y)-Y_{\rho}^{\partial \psi}(x)\right\rangle \geq \psi\left(Y_{\rho}^{\partial \psi}(y)\right)-\psi\left(Y_{\rho}^{\partial \psi}(x)\right), \\
& \left\langle N(u, v, w), Y_{\rho}^{\partial \psi}(y)-Y_{\rho}^{\partial \psi}(x)\right\rangle \oplus\left(\psi\left(Y_{\rho}^{\partial \psi}(y)\right)-\psi\left(Y_{\rho}^{\partial \psi}(x)\right)\right) \geq 0,
\end{aligned}
$$

which is the Yosida variational inequality problem involving XOR-operation (22).

On the other hand, let the Yosida variational inequality problem (22) holds. That is, $x \in \mathscr{C}, u \in A(x), v \in$ $B(x), w \in C(x)$ such that $\left\langle N(u, v, w), Y_{\rho}^{\partial \psi}(y)-Y_{\rho}^{\partial \psi}(x)\right\rangle \oplus\left(\psi\left(Y_{\rho}^{\partial \psi}(y)\right)-\psi\left(Y_{\rho}^{\partial \psi}(x)\right)\right)$ $\geq 0, \quad \forall y \in \mathscr{C}$. 
As $\mathscr{C}$ is a closed convex pointed cone, $y=2 x \in \mathscr{C}$ as well as $y=(1 / 2) x \in \mathscr{C}$. Putting $y=2 x$ and $y=(1 / 2) x$ and using linearity of $\psi$ and Proposition 3, we have

$$
\begin{aligned}
& \left\langle N(u, v, w), Y_{\rho}^{\partial \psi}(2 x)-Y_{\rho}^{\partial \psi}(x)\right\rangle \oplus\left(\psi\left(Y_{\rho}^{\partial \psi}(2 x)\right)-\psi\left(Y_{\rho}^{\partial \psi}(x)\right)\right) \geq 0 \\
& \left\langle N(u, v, w), 2 Y_{\rho}^{\partial \psi}(x)-Y_{\rho}^{\partial \psi}(x)\right\rangle \oplus\left(\psi\left(2 Y_{\rho}^{\partial \psi}(x)\right)-\psi\left(Y_{\rho}^{\partial \psi}(x)\right)\right) \geq 0 \\
& \left\langle N(u, v, w), Y_{\rho}^{\partial \psi}(x)\right\rangle \oplus\left(2 \psi\left(Y_{\rho}^{\partial \psi}(x)\right)-\psi\left(Y_{\rho}^{\partial \psi}(x)\right)\right) \geq 0 \\
& \left\langle N(u, v, w), Y_{\rho}^{\partial \psi}(x)\right\rangle \oplus \psi\left(Y_{\rho}^{\partial \psi}(x)\right) \geq 0, \\
& \left\langle N(u, v, w), Y_{\rho}^{\partial \psi}\left(\frac{1}{2} x\right)-Y_{\rho}^{\partial \psi}(x)\right\rangle \oplus\left(\psi\left(Y_{\rho}^{\partial \psi}\left(\frac{1}{2} x\right)\right)-\psi\left(Y_{\rho}^{\partial \psi}(x)\right)\right) \geq 0 \\
& \left\langle N(u, v, w), \frac{-1}{2} Y_{\rho}^{\partial \psi}(x)\right\rangle \oplus\left(\frac{-1}{2} \psi\left(Y_{\rho}^{\partial \psi}(x)\right)\right) \geq 0 \\
& \left\langle N(u, v, w), \frac{-1}{2} Y_{\rho}^{\partial \psi}(x)\right\rangle \geq \frac{-1}{2}\left(\psi\left(Y_{\rho}^{\partial \psi}(x)\right)\right) \\
& \left\langle N(u, v, w), Y_{\rho}^{\partial \psi}(x)\right\rangle \leq \psi\left(Y_{\rho}^{\partial \psi}(x)\right) .
\end{aligned}
$$

Thus, we have

$$
\left\langle N(u, v, w), Y_{\rho}^{\partial \psi}(x)\right\rangle \oplus \psi\left(Y_{\rho}^{\partial \psi}(x)\right) \leq 0 .
$$

Adding (31) and (32), we have

$$
\left\langle N(u, v, w), Y_{\rho}^{\partial \psi}(x)\right\rangle \oplus \psi\left(Y_{\rho}^{\partial \psi}(x)\right)=0 .
$$

Since

$$
\left\langle N(u, v, w), Y_{\rho}^{\partial \psi}(y)-Y_{\rho}^{\partial \psi}(x)\right\rangle \oplus\left(\psi\left(Y_{\rho}^{\partial \psi}(y)\right)-\psi\left(Y_{\rho}^{\partial \psi}(x)\right)\right) \geq 0,
$$

we have

$$
\begin{aligned}
& \left\langle N(u, v, w), Y_{\rho}^{\partial \psi}(y)\right\rangle-\left\langle N(u, v, w), Y_{\rho}^{\partial \psi}(x)\right\rangle \\
& \geq \psi\left(Y_{\rho}^{\partial \psi}(y)\right)-\psi\left(Y_{\rho}^{\partial \psi}(x)\right) .
\end{aligned}
$$

Using (25), from the above inequality, we have

$$
\left\langle N(u, v, w), Y_{\rho}^{\partial \psi}(y)\right\rangle-\psi\left(Y_{\rho}^{\partial \psi}(x)\right) \geq \psi\left(Y_{\rho}^{\partial \psi}(y)\right)-\psi\left(Y_{\rho}^{\partial \psi}(x)\right),
$$

it follows that

$$
\left\langle N(u, v, w), Y_{\rho}^{\partial \psi}(y)\right\rangle \geq \psi\left(Y_{\rho}^{\partial \psi}(y)\right)
$$

Using (ii) of Proposition 1, we have

$$
\begin{gathered}
\left\langle N(u, v, w), Y_{\rho}^{\partial \psi}(y)\right\rangle \oplus \psi\left(Y_{\rho}^{\partial \psi}(y)\right) \\
\geq \psi\left(Y_{\rho}^{\partial \psi}(y)\right) \oplus \psi\left(Y_{\rho}^{\partial \psi}(y)\right), \\
\left\langle N(u, v, w), Y_{\rho}^{\partial \psi}(y)\right\rangle \oplus \psi\left(Y_{\rho}^{\partial \psi}(y)\right) \geq 0 .
\end{gathered}
$$

Combination of (33) and (39) is the required Yosida complementarity problem involving XOR-operation (21).

The following Lemma guarantees the equivalence between the Yosida variational inequality problem involving XOR-operation (22) and a fixed point equation.

Lemma 2. Let $P: \mathscr{C} \longrightarrow \mathscr{C}$ be a mapping, then the Yosida variational inequality problem involving XOR-operation (22) has a solution $x \in \mathscr{C}, u \in A(x), v \in B(x), w \in C(x)$, if and only if it satisfies the equation:

$$
Y_{\rho}^{\partial \psi}(x)=J_{\rho}^{\partial \psi}\left[P\left(Y_{\rho}^{\partial \psi}(x)\right)+\rho N(u, v, w)\right],
$$

where $\rho>0$ is a constant.

Proof. Let $\quad x \in \mathscr{C}, u \in A(x), v \in B(x), w \in C(x)$ satisfy equation (40), that is,

$$
Y_{\rho}^{\partial \psi}(x)=J_{\rho}^{\partial \psi}\left[P\left(Y_{\rho}^{\partial \psi}(x)\right)+\rho N(u, v, w)\right] .
$$

Using the definition of the proximal operator $J_{\rho}^{\partial \psi}$ and from the above equation, we have 


$$
\begin{aligned}
Y_{\rho}^{\partial \psi}(x)= & {[P+\rho \partial \psi]^{-1}\left[P\left(Y_{\rho}^{\partial \psi}(x)\right)+\rho N(u, v, w)\right] } \\
P\left(Y_{\rho}^{\partial \psi}(x)\right)+\rho \partial \psi\left(Y_{\rho}^{\partial \psi}(x)\right)= & P\left(Y_{\rho}^{\partial \psi}(x)\right)+\rho N(u, v, w) \quad \text { (that is, ) } \\
& N(u, v, w) \in \partial \psi\left(Y_{\rho}^{\partial \psi}(x)\right) . \quad \text { (which gives us) }
\end{aligned}
$$

Applying the definition of subdifferential operator, the above inclusion holds if and only if

$\psi\left(Y_{\rho}^{\partial \psi}(y)\right)-\psi\left(Y_{\rho}^{\partial \psi}(x)\right) \geq\left\langle N(u, v, w), \psi\left(Y_{\rho}^{\partial \psi}(y)\right)-\psi\left(Y_{\rho}^{\partial \psi}(x)\right)\right\rangle$.
Using (ii) of Proposition 1, we have

$$
\begin{aligned}
& \left\langle N(u, v, w), \psi\left(Y_{\rho}^{\partial \psi}(y)\right)-\psi\left(Y_{\rho}^{\partial \psi}(x)\right)\right\rangle \oplus\left(\psi\left(Y_{\rho}^{\partial \psi}(y)\right)-\psi\left(Y_{\rho}^{\partial \psi}(x)\right)\right) \\
& \geq\left\langle N(u, v, w), \psi\left(Y_{\rho}^{\partial \psi}(y)\right)-\psi\left(Y_{\rho}^{\partial \psi}(x)\right)\right\rangle \oplus\left\langle N(u, v, w), \psi\left(Y_{\rho}^{\partial \psi}(y)\right)-\psi\left(Y_{\rho}^{\partial \psi}(x)\right)\right\rangle .
\end{aligned}
$$

It follows that

$$
\left\langle N(u, v, w), Y_{\rho}^{\partial \psi}(y)-Y_{\rho}^{\partial \psi}(x)\right\rangle \oplus\left(\psi\left(Y_{\rho}^{\partial \psi}(y)\right)-\psi\left(Y_{\rho}^{\partial \psi}(x)\right)\right)
$$$$
\geq 0, \quad \forall y \in \mathscr{C},
$$

which is the required Yosida variational inequality problem involving XOR-operation (22).

The Lemma mentioned below ensures the equivalence between the Yosida variational inequality problem involving XOR-operation (22) and the Yosida proximal operator equation involving XOR-operation (23).

Lemma 3. Suppose $N(u, v, w) \propto R_{\rho}^{\partial \psi}(z)$ and $P: \mathscr{C} \longrightarrow \mathscr{C}$ is a one-one mapping. Then $x \in \mathscr{C}, u \in A(x), v \in B(x)$, $w \in C(x)$ is the solution of the Yosida variational inequality problem involving XOR-operation (22) if and only if $x, z \in \mathscr{C}, u \in A(x), v \in B(x), w \in C(x)$ satisfy the Yosida proximal operator equation involving XOR-operation (23), where $R_{\rho}^{\partial \psi}=\left[I-P\left(J_{\rho}^{\partial \psi}\right)\right]$, in which $J_{\rho}^{\partial \psi}$ is the proximal operator and $P\left(J_{\rho}^{\partial \psi}(z)\right)=P\left(J_{\rho}^{\partial \psi}\right)(z)$.

Proof. Let $x \in \mathscr{C}, u \in A(x), v \in B(x), w \in C(x)$ be the solution of the Yosida variational inequality problem involving XOR-operation (22). Then by Lemma 2, it satisfies the equation:

$$
Y_{\rho}^{\partial \psi}(x)=J_{\rho}^{\partial \psi}\left[P\left(Y_{\rho}^{\partial \psi}(x)\right)+\rho N(u, v, w)\right] .
$$

Let $z=P\left(Y_{\rho}^{\partial \psi}(x)\right)+\rho N(u, v, w)$, then

$$
\begin{aligned}
Y_{\rho}^{\partial \psi}(x) & =J_{\rho}^{\partial \psi}(z), \\
\operatorname{As} z & =P\left(J_{\rho}^{\partial \psi}(z)\right)+\rho N(u, v, w) \\
z-P\left(J_{\rho}^{\partial \psi}(z)\right) & =\rho N(u, v, w) \\
\left(I-P\left(J_{\rho}^{\partial \psi}\right)\right)(z) & =\rho N(u, v, w) \\
R_{\rho}^{\partial \psi}(z) & =\rho N(u, v, w) \\
\rho^{-1} R_{\rho}^{\partial \psi}(z) & =N(u, v, w), \text { where } P\left(J_{\rho}^{\partial \psi}(z)\right)=P\left(J_{\rho}^{\partial \psi}\right)(z) .
\end{aligned}
$$

Using (ii) of Proposition 1, we have

$$
N(u, v, w) \oplus \rho^{-1} R_{\rho}^{\partial \psi}(z)=N(u, v, w) \oplus N(u, v, w)=0 .
$$

Thus, we have

$$
N(u, v, w) \oplus \rho^{-1} R_{\rho}^{\partial \psi}(z)=0,
$$

which is the required Yosida proximal operator equation involving XOR-operation (23).

Conversely, let $x, z \in \mathscr{C}, u \in A(x), v \in B(x), w \in C(x)$ be the solution of Yosida proximal operator equation involving XOR-operation (23).

That is, we have

$$
N(u, v, w) \oplus \rho^{-1} R_{\rho}^{\partial \psi}(z)=0 .
$$

Using (v) of Proposition 1, definition of $R_{\rho}^{\partial \psi}$ and comparability of $N(u, v, w)$ with $R_{\rho}^{\partial \psi}(z)$, we obtain

$$
\begin{aligned}
\rho N(u, v, w) & =R_{\rho}^{\partial \psi}(z)=\left[I-P\left(J_{\rho}^{\partial \psi}\right)\right](z) \\
& =z-P\left(J_{\rho}^{\partial \psi}\right)(z) \\
& =P\left(Y_{\rho}^{\partial \psi}(x)\right)+\rho N(u, v, w)-P\left(J_{\rho}^{\partial \psi}\left(\left(P\left(Y_{\rho}^{\partial \psi}(x)\right)+\rho N(u, v, w)\right)\right) .\right.
\end{aligned}
$$


From above, we have

$P\left(Y_{\rho}^{\partial \psi}(x)\right)=P\left(J_{\rho}^{\partial \psi}\left(P\left(Y_{\rho}^{\partial \psi}(x)\right)+\rho N(u, v, w)\right)\right)$.

Since $P$ is a one-one mapping, we obtain

$$
Y_{\rho}^{\partial \psi}(x)=J_{\rho}^{\partial \psi}\left[P\left(Y_{\rho}^{\partial \psi}(x)\right)+\rho N(u, v, w)\right] .
$$

Applying Lemma 2, we conclude that $x \in \mathscr{C}, u \in A(x), v \in B(x), w \in C(x)$ is the solution of Yosida variational inequality problem involving XOR-operation (22).

\section{Algorithm and Existence Results}

Invoking Lemmas 2 and 3, we suggest the following algorithm for solving Yosida proximal operator equation involving XOR-operation (23).

Algorithm 1. For any $x_{0}, z_{0} \in \mathscr{C}, u_{0} \in A\left(x_{0}\right), v_{0} \in B\left(x_{0}\right)$, $w_{0} \in C\left(x_{0}\right)$, we let

$$
z_{1}=P\left(Y_{\rho}^{\partial \psi}\left(x_{0}\right)\right)+\rho N\left(u_{0}, v_{0}, w_{0}\right)
$$

Take any $x_{1} \in \mathscr{C}$ such that

$$
Y_{\rho}^{\partial \psi}\left(x_{1}\right)=J_{\rho}^{\partial \psi}\left(z_{1}\right)
$$

Since $u_{0} \in A\left(x_{0}\right), v_{0} \in B\left(x_{0}\right), w_{0} \in C\left(x_{0}\right)$, by Nadler's theorem [38], there exist $u_{1} \in A\left(x_{1}\right), v_{0} \in B\left(x_{1}\right)$, $w_{0} \in C\left(x_{1}\right)$, using (viii) of Proposition 1 and comparability of $u_{0}, u_{1} ; v_{0}, v_{1}$ and $w_{0}, w_{1}$, we have

$$
\begin{gathered}
\left\|u_{0} \oplus u_{1}\right\|=\left\|u_{0}-u_{1}\right\| \leq(1+1) D\left(A\left(Y_{\rho}^{\partial \psi}\left(x_{0}\right)\right), A\left(Y_{\rho}^{\partial \psi}\left(x_{1}\right)\right)\right), \\
\left\|v_{0} \oplus v_{1}\right\|=\left\|v_{0}-v_{1}\right\| \leq(1+1) D\left(B\left(Y_{\rho}^{\partial \psi}\left(x_{0}\right)\right), B\left(Y_{\rho}^{\partial \psi}\left(x_{1}\right)\right)\right), \\
\left\|w_{0} \oplus w_{1}\right\|=\left\|w_{0}-w_{1}\right\| \leq(1+1) D\left(C\left(Y_{\rho}^{\partial \psi}\left(x_{0}\right)\right), C\left(Y_{\rho}^{\partial \psi}\left(x_{1}\right)\right)\right),
\end{gathered}
$$

where $D(\cdot, \cdot)$ is the Hausdorff metric on $\widetilde{C} B(H)$.

Let $z_{2}=P\left(Y_{\rho}^{\partial \psi}\left(x_{1}\right)\right)+\rho N\left(u_{1}, v_{1}, w_{1}\right)$ and take any $x_{2} \in \mathscr{C}$ such that

$$
Y_{\rho}^{\partial \psi}\left(x_{2}\right)=J_{\rho}^{\partial \psi}\left(z_{2}\right)
$$

Continuing the above procedure, we compute the sequences $\left\{x_{n}\right\},\left\{u_{n}\right\},\left\{v_{n}\right\}$ and $\left\{z_{n}\right\}$ by the schemes given below:

$$
\begin{aligned}
Y_{\rho}^{\partial \psi}\left(x_{n}\right) & =J_{\rho}^{\partial \psi}\left(z_{n}\right), \\
u_{n} & \in A\left(x_{n}\right), u_{n+1} \in A\left(x_{n+1}\right) \text { such that } u_{n} \propto u_{n+1}, \\
\left\|u_{n} \oplus u_{n+1}\right\| & =\left\|u_{n}-u_{n+1}\right\| \leq\left(1+\frac{1}{n+1}\right) D\left(A\left(Y_{\rho}^{\partial \psi}\left(x_{n}\right)\right), A\left(Y_{\rho}^{\partial \psi}\left(x_{n+1}\right)\right)\right), \\
v_{n} & \in B\left(x_{n}\right), v_{n+1} \in B\left(x_{n+1}\right) \text { such that } v_{n} \propto v_{n+1}, \\
\left\|v_{n} \oplus v_{n+1}\right\| & =\left\|v_{n}-v_{n+1}\right\| \leq\left(1+\frac{1}{n+1}\right) D\left(B\left(Y_{\rho}^{\partial \psi}\left(x_{n}\right)\right), B\left(Y_{\rho}^{\partial \psi}\left(x_{n+1}\right)\right)\right), \\
w_{n} & \in C\left(x_{n}\right), w_{n+1} \in C\left(x_{n+1}\right) \text { such that } w_{n} \propto w_{n+1}, \\
\left\|w_{n} \oplus w_{n+1}\right\| & =\left\|w_{n}-w_{n+1}\right\| \leq\left(1+\frac{1}{n+1}\right) D\left(C\left(Y_{\rho}^{\partial \psi}\left(x_{n}\right)\right), C\left(Y_{\rho}^{\partial \psi}\left(x_{n+1}\right)\right)\right), \\
z_{n+1} & =P\left(Y_{\rho}^{\partial \psi}\left(x_{n}\right)\right)+\rho N\left(u_{n}, v_{n}, w_{n}\right),
\end{aligned}
$$

where $\rho>0$ is a constant and $n=0,1,2,3, \cdots$.

Theorem 1. Let $H$ be a real ordered positive Hilbert Space and $\mathscr{C} \subseteq H$ be a closed convex pointed cone. Let $A, B, C: \mathscr{C} \longrightarrow \widetilde{C} B(H)$ be the D-Lipschitz continuous mappings with constants $\lambda_{D_{A}}, \lambda_{D_{B}}$, and $\lambda_{D_{C}}$, respectively. Let $N: H \times H \times H \longrightarrow H$ be a single-valued mapping such that $N$ is Lipschitz continuous in first, second, and third arguments with constants $\lambda_{N_{1}}, \lambda_{N_{2}}$, and $\lambda_{N_{3}}$, respectively. Let $Y_{\rho}^{\partial \psi}: \mathscr{C} \longrightarrow \mathscr{C}$ be the Yosida approximation operator such that $Y_{\rho}^{\partial \psi}$ is strongly monotone with constant $\delta_{y}$ and $J_{\rho}^{\partial \psi}: \mathscr{C} \longrightarrow \mathscr{C}$ be the proximal operator such that $J_{\rho}^{\partial \psi}$ is Lipschitz continuous with constant $\theta$. Suppose $P: \mathscr{C} \longrightarrow \mathscr{C}$ be a Lipschitz continuous mapping with constant $\lambda_{P}$, strongly monotone with respect to $J_{\rho}^{\partial \psi}$ with constant $\mu$ and $\psi: H \longrightarrow \mathbb{R} \cup\{+\infty\}$ be a strongly convex, subdifferentiable, proper functional satisfying $Y_{\rho}^{\partial \psi}(x) \in$ do $m(\partial \psi)$. Suppose that $z_{n+1} \propto z_{n}$, for $n=0,1,2, \cdots$ and if the following condition is satisfied:

$$
\begin{aligned}
& \theta<2 \delta_{y}, \xi(\theta)<\frac{1-\lambda_{P} \theta}{\rho \theta}, \quad \text { where } \xi(\theta) \\
& =\lambda_{N_{1}} \lambda_{D_{A}}+\lambda_{N_{2}} \lambda_{D_{B}}+\lambda_{N_{3}} \lambda_{D_{C}} \text { and } \theta=\frac{1}{\sqrt{\sigma \rho+\mu}},
\end{aligned}
$$

then there exists $x, z \in \mathscr{C}, u \in A(x), v \in B(x), w \in C(x)$ satisfying the Yosida proximal operator equation involving XOR-operation (23) and the sequences $\left\{x_{n}\right\},\left\{z_{n}\right\},\left\{u_{n}\right\},\left\{v_{n}\right\}$ 
and $\left\{w_{n}\right\}$ generated by Algorithm 1 converge strongly to $x, z, u, v$, and $w$, respectively.
Proof. Using (x) of Algorithm 1 and (ii) of Proposition 1, we have

$$
\begin{aligned}
0 & \leq z_{n+1} \oplus z_{n} \\
& =\left[P\left(Y_{\rho}^{\partial \psi}\left(x_{n}\right)\right)+\rho N\left(u_{n}, v_{n}, w_{n}\right)\right] \oplus\left[P\left(Y_{\rho}^{\partial \psi}\left(x_{n}\right)\right)+\rho N\left(u_{n-1}, v_{n-1}, w_{n-1}\right)\right] \\
& =\left[P\left(Y_{\rho}^{\partial \psi}\left(x_{n}\right)\right) \oplus P\left(Y_{\rho}^{\partial \psi}\left(x_{n-1}\right)\right)\right]+\rho\left[N\left(u_{n}, v_{n}, w_{n}\right) \oplus N\left(u_{n-1}, v_{n-1}, w_{n-1}\right)\right] .
\end{aligned}
$$

It follows from (60) that

$$
\begin{aligned}
\left\|z_{n+1} \oplus z_{n}\right\| \leq & \left\|P\left(Y_{\rho}^{\partial \psi}\left(x_{n}\right)\right) \oplus P\left(Y_{\rho}^{\partial \psi}\left(x_{n-1}\right)\right)\right\| \\
& +\rho\left\|N\left(u_{n}, v_{n}, w_{n}\right) \oplus N\left(u_{n-1}, v_{n-1}, w_{n-1}\right)\right\| .
\end{aligned}
$$

Since $z_{n+1} \propto z_{n}$ and using (vii) and (viii) of Proposition 1 , from (61), we obtain

$$
\begin{aligned}
\left\|z_{n+1}-z_{n}\right\| \leq & \left\|P\left(Y_{\rho}^{\partial \psi}\left(x_{n}\right)\right)-P\left(Y_{\rho}^{\partial \psi}\left(x_{n-1}\right)\right)\right\| \\
& +\rho\left\|N\left(u_{n}, v_{n}, w_{n}\right)-N\left(u_{n-1}, v_{n-1}, w_{n-1}\right)\right\| .
\end{aligned}
$$

Since $N$ is Lipschitz continuous in all the three arguments with constants $\lambda_{N_{1}}, \lambda_{N_{2}}$, and $\lambda_{N_{3}}$, respectively, and $A, B, C$ are $D$-Lipschitz continuous mappings with constants $\lambda_{D_{A}}, \lambda_{D_{B}}, \lambda_{D_{C}}$, respectively, and using (vii), (viii), (ix) of Algorithm 1, we have

$$
\begin{aligned}
\| & N\left(u_{n}, v_{n}, w_{n}\right)-N\left(u_{n-1}, v_{n-1}, w_{n-1}\right)\|=\| N\left(u_{n}, v_{n}, w_{n}\right)-N\left(u_{n-1}, v_{n}, w_{n}\right)+N\left(u_{n-1}, v_{n}, w_{n}\right) \\
& -N\left(u_{n-1}, v_{n-1}, w_{n}\right)+N\left(u_{n-1}, v_{n-1}, w_{n}\right)-N\left(u_{n-1}, v_{n-1}, w_{n-1}\right) \| \\
\leq & \lambda_{N_{1}}\left\|u_{n}-u_{n-1}\right\|+\lambda_{N_{2}}\left\|v_{n}-v_{n-1}\right\| \lambda_{N_{3}}\left\|w_{n}-w_{n-1}\right\| \\
\leq & \lambda_{N_{1}}\left[\left(1+\frac{1}{n}\right) D\left(A\left(Y_{\rho}^{\partial \psi}\left(x_{n}\right)\right), A\left(Y_{\rho}^{\partial \psi}\left(x_{n-1}\right)\right)\right)\right] \\
& +\lambda_{N_{2}}\left[\left(1+\frac{1}{n}\right) D\left(B\left(Y_{\rho}^{\partial \psi}\left(x_{n}\right)\right), B\left(Y_{\rho}^{\partial \psi}\left(x_{n-1}\right)\right)\right)\right] \\
& +\lambda_{N_{3}}\left[\left(1+\frac{1}{n}\right) D\left(C\left(Y_{\rho}^{\partial \psi}\left(x_{n}\right)\right), C\left(Y_{\rho}^{\partial \psi}\left(x_{n-1}\right)\right)\right)\right] \\
\leq & {\left[\lambda_{N_{1}}\left(1+\frac{1}{n}\right) \lambda_{D_{A}}\left\|Y_{\rho}^{\partial \psi}\left(x_{n}\right)-Y_{\rho}^{\partial \psi}\left(x_{n-1}\right)\right\|\right] } \\
& +\left[\lambda_{N_{2}}\left(1+\frac{1}{n}\right) \lambda_{D_{B}}\left\|Y_{\rho}^{\partial \psi}\left(x_{n}\right)-Y_{\rho}^{\partial \psi}\left(x_{n-1}\right)\right\|\right] \\
& +\left[\lambda_{N_{3}}\left(1+\frac{1}{n}\right) \lambda_{D_{C}}\left\|Y_{\rho}^{\partial \psi}\left(x_{n}\right)-Y_{\rho}^{\partial \psi}\left(x_{n-1}\right)\right\|\right] \\
= & \left(\lambda_{N_{1}} \lambda_{D_{A}}+\lambda_{N_{2}} \lambda_{D_{B}}+\lambda_{N_{3}} \lambda_{D_{C}}\right)\left(1+\frac{1}{n}\right)\left\|Y_{\rho}^{\partial \psi}\left(x_{n}\right)-Y_{\rho}^{\partial \psi}\left(x_{n-1}\right)\right\| .
\end{aligned}
$$

Using strong monotonicity of the Yosida approximation operator $Y_{\rho}^{\partial \psi}$ with constant $\delta_{Y}$ and Lipschitz continuity of the proximal operator $J_{\rho}^{\partial \psi}$ with constant $\theta$, we have

$$
\begin{aligned}
\left\|x_{n}-x_{n-1}\right\|^{2} & =\left\|J_{\rho}^{\partial \psi}\left(z_{n}\right)-J_{\rho}^{\partial \psi}\left(z_{n-1}\right)-\left[Y_{\rho}^{\partial \psi}\left(x_{n}\right)-x_{n}-\left(Y_{\rho}^{\partial \psi}\left(x_{n-1}\right)-x_{n-1}\right)\right]\right\|^{2} \\
& \leq\left\|J_{\rho}^{\partial \psi}\left(z_{n}\right)-J_{\rho}^{\partial \psi}\left(z_{n-1}\right)\right\|^{2}-2\left\langle Y_{\rho}^{\partial \psi}\left(x_{n}\right)-Y_{\rho}^{\partial \psi}\left(x_{n-1}\right), x_{n}-x_{n-1}\right\rangle+\left\|x_{n}-x_{n-1}\right\|^{2} \\
& \leq \theta^{2}\left\|z_{n}-z_{n-1}\right\|^{2}-2 \delta_{y}\left\|x_{n}-x_{n-1}\right\|^{2}+\left\|x_{n}-x_{n-1}\right\|^{2} .
\end{aligned}
$$


It follows that

$$
\begin{aligned}
2 \delta_{y}\left\|x_{n}-x_{n-1}\right\|^{2} & \leq \theta^{2}\left\|z_{n}-z_{n-1}\right\|^{2}, \\
\left\|x_{n}-x_{n-1}\right\| & \leq \frac{\theta^{2}}{\sqrt{2 \delta_{y}}}\left\|z_{n}-z_{n-1}\right\|, \\
\left\|x_{n}-x_{n-1}\right\| & \leq \xi(y)\left\|z_{n}-z_{n-1}\right\|,
\end{aligned}
$$

where $\xi(y)=\theta^{2} / \sqrt{2 \delta_{y}}$.

Combining (62) and (63), using Lipschitz continuity of $P, J_{\rho}^{\partial \psi}$ and (vi) of Algorithm 1, we have

$$
\begin{aligned}
\left\|z_{n+1}-z_{n}\right\| \leq & \lambda_{P}\left\|\left(Y_{\rho}^{\partial \psi}\left(x_{n}\right)\right)-\left(Y_{\rho}^{\partial \psi}\left(x_{n-1}\right)\right)\right\| \\
& +\rho\left(\lambda_{N_{1}} \lambda_{D_{A}}+\lambda_{N_{2}} \lambda_{D_{B}}+\lambda_{N_{3}} \lambda_{D_{C}}\right)\left(1+\frac{1}{n}\right)\left\|Y_{\rho}^{\partial \psi}\left(x_{n}\right)-Y_{\rho}^{\partial \psi}\left(x_{n-1}\right)\right\| \\
\left\|z_{n+1}-z_{n}\right\| \leq & {\left[\lambda_{P}+\rho \xi_{n}(\theta)\right]\left\|Y_{\rho}^{\partial \psi}\left(x_{n}\right)-Y_{\rho}^{\partial \psi}\left(x_{n-1}\right)\right\| } \\
\leq & {\left[\lambda_{P}+\rho \xi_{n}(\theta)\right]\left\|J_{\rho}^{\partial \psi}\left(z_{n}\right)-J_{\rho}^{\partial \psi}\left(z_{n-1}\right)\right\| } \\
= & {\left[\lambda_{P}+\rho \xi_{n}(\theta)\right] \theta\left\|z_{n}-z_{n-1}\right\| } \\
= & {\left[\lambda_{P} \theta+\rho \xi_{n}(\theta) \theta\right]\left\|z_{n}-z_{n-1}\right\| } \\
= & S_{n}(\theta)\left\|z_{n}-z_{n-1}\right\|
\end{aligned}
$$

where $\quad S_{n}(\theta)=\lambda_{P} \theta+\rho \xi_{n}(\theta) \theta, \theta=(1 / \sqrt{\sigma \rho+\mu})$

and $\xi_{n}(\theta)=\left(\lambda_{N_{1}} \lambda_{D}+\lambda_{N_{2}} \lambda_{D_{B}}+\lambda_{N_{3}} \lambda_{D_{C}}\right)(1+(1 / n))$.

Letting $S(\theta)=\lambda_{P} \theta+\rho \xi(\theta) \theta$, where $\xi(\theta)=\left(\lambda_{N_{1}} \lambda_{D_{A}}+\right.$ $\left.\lambda_{N_{2}} \lambda_{D_{B}}+\lambda_{N_{3}} \lambda_{D_{C}}\right)$, it follows that $S_{n}(\theta) \longrightarrow S(\theta)$ as $n \longrightarrow \infty$. From (59), we have $\xi(y)<1$ and $S(\theta)<1$. Consequently, we conclude from (65) and (67) that $\left\{x_{n}\right\}$ and $\left\{z_{n}\right\}$ both are Cauchy sequences. Since $H$ is complete and $\mathscr{C} \subseteq H$ is a closed convex subset of $H$ and thus $\mathscr{C}$ is also complete, we may assume that $x_{n} \longrightarrow x \in \mathscr{C}$ and $z_{n} \longrightarrow z \in \mathscr{C}$. From (vii), (viii), and (ix) of Algorithm 1, it follows that $\left\{u_{n}\right\},\left\{v_{n}\right\}$, and $\left\{w_{n}\right\}$ are also Cauchy sequences such that $u_{n} \longrightarrow u, v_{n} \longrightarrow v$ and $w_{n} \longrightarrow w$, as $n \longrightarrow \infty$.

It can be shown easily by using the techniques of [28] that $u \in A(x), v \in B(x)$, and $w \in C(x)$. By Lemma 3 , we conclude that $x, z \in \mathscr{C}, u \in A(x), v \in B(x)$, and $w \in C(x)$ is the solution of Yosida proximal operator equation involving XOR-operation (23).

We provide the following numerical example using MATLAB program R2018a along with a computational table and a convergence graphs for different initial values in support of Algorithm 1 and Theorem 1.

Example 1. Suppose $\mathscr{C}=H=[0, \infty)$. Let $P: \mathscr{C} \longrightarrow \mathscr{C}$ and $\psi: \mathscr{C} \longrightarrow \mathbb{R} \cup\{+\infty\}$ be the mappings such that for $x \in \mathscr{C}$,

$$
\begin{aligned}
& P(x)=\frac{x}{2}, \\
& \psi(x)=x^{2},
\end{aligned}
$$

Then $\partial \psi(x)=\{2 x\}$, the sub differential of $\psi$.
Since $\psi^{\prime \prime}(x)=2>0$. Hence, $\psi$ is strongly convex with modulus $\lambda=2$.

For $\rho=1$, the proximal operator $J_{\rho}^{\partial \psi}$ is given by

$J_{\rho}^{\partial \psi}=[P+\rho \partial \psi]^{-1}(x)=\frac{2 x}{5}$, where $[P+\rho \partial \psi](x)=\frac{5 x}{2}$.

It is simple to see that $P$ is Lipschitz continuous with constant $\lambda_{p}=(11 / 10)$, strongly monotone with respect to $J_{\rho}^{\partial \psi}$ with constant $\mu=1 / 3$, and $J_{\rho}^{\partial \psi}$ is Lipschitz continuous with constant $\theta=\sqrt{2} / 3$.

In view of proximal operator calculated above, the Yosida approximation operator is given by

$$
Y_{\rho}^{\partial \psi}(x)=\frac{1}{\rho}\left[I-J_{\rho}^{\partial \psi}\right](x)=\frac{3 x}{5} .
$$

Also,

$$
\begin{aligned}
\left\langle Y_{\rho}^{\partial \psi}(x)-Y_{\rho}^{\partial \psi}(y), x-y\right\rangle & =\left\langle\frac{3 x}{5}-\frac{3 y}{5}, x-y\right\rangle \\
& =\left\langle\frac{3}{5}(x-y), x-y\right\rangle \\
& =\frac{3}{5}\langle x-y, x-y\rangle=\frac{3}{5}\|x-y\|^{2} \\
& \geq \frac{2}{5}\|x-y\|^{2} .
\end{aligned}
$$

Hence, $Y_{\rho}^{\partial \psi}$ is strongly monotone with constant $\delta_{y}=(2 / 5)$. 
Let us consider the mappings $N: H \times H \times H \longrightarrow H$ and $A, B, C: \mathscr{C} \longrightarrow \widetilde{C} B(H)$ such that

$$
\begin{aligned}
A(x) & =\left\{\frac{x}{7}\right\}, \\
B(x) & =\left\{\frac{x}{5}\right\}, \\
C(x) & =\left\{\frac{x}{6}\right\}, \\
N(u, v, w) & =\frac{u}{2}+\frac{v}{2}+\frac{w}{2},
\end{aligned}
$$

where $x \in \mathscr{C}, u \in A(x), v \in B(x)$, and $w \in C(x)$.

that is, $D(A(x), A(y)) \leq(1 / 5)\|x-y\|$.

Thus, $A$ is $D$-Lipschitz continuous with constant $\lambda_{D_{A}}=(1 / 5)$. Similarly, we can obtain that $B$ and $C$ are $D$ Lipschitz continuous with constants $\lambda_{D_{B}}=(1 / 3)$ and $\lambda_{D_{C}}=(1 / 3)$, respectively.

$N$ is Lipschitz continuous in all the three arguments with constants $\lambda_{N_{1}}=\lambda_{N_{2}}=\lambda_{N_{3}}=1$.

$$
\text { Then, } \begin{aligned}
N(u, v, w) & =\frac{x}{14}+\frac{x}{10}+\frac{x}{12} \\
& =\frac{107 x}{420}, \\
\text { Since } z & =P\left(Y_{\rho}^{\partial \psi}(x)\right)+\rho N(u, v, w) \\
& =\frac{Y_{\rho}^{\partial \psi}(x)}{2}+N(u, v, w) \\
& =\frac{1}{2} \cdot \frac{3 x}{5}+N(u, v, w), \\
z & =\frac{3 x}{10}+N(u, v, w), \\
J_{\rho}^{\partial \psi}(z) & =\frac{6 x}{50}+\frac{2 N(u, v, w)}{5} .
\end{aligned}
$$

Hence,

$$
R_{\rho}^{\partial \psi}(z)=\left[I-J_{\rho}^{\partial \psi}\right](z)=\frac{3}{5}\left[\frac{3 x}{10}+N(u, v, w)\right] .
$$

Below we show that condition (59) is satisfied.
For $\lambda_{P}=11 / 10, \rho=1, \delta_{y}=2 / 5, \mu=1 / 3, \lambda=2, \sigma=2 \lambda=$ $4, \theta=\sqrt{2} / 3, \lambda_{N_{1}}=\lambda_{N_{2}}=\lambda_{N_{3}}=1, \lambda_{D_{A}}=1 / 5, \lambda_{D_{B}}=1 / 3, \lambda_{D_{C}}=$ $1 / 3, \xi(\theta)=\lambda_{N_{1}} \lambda_{D_{A}}+\lambda_{N_{2}} \lambda_{D_{B}}+\lambda_{N_{3}} \lambda_{D_{C}}=0.86$ and $\left(1-\lambda_{P}\right.$ $\theta) / \rho \theta=1.021$. Hence, $\theta<2 \delta_{y}$ and $\xi(\theta)<\left(1-\lambda_{P} \theta\right) / \rho \theta$. That is, condition (59) is satisfied.

For, $x=0$, the Yosida proximal operator equation involving XOR-operator (23) is fulfilled.

$$
\begin{aligned}
& \text { That is, } N(u, v, w) \oplus \rho^{-1} R_{\rho}^{\partial \psi}(z) \\
& =N(u, v, w) \oplus \frac{3}{5}\left[\frac{3 x}{10}+N(u, v, w)\right]=0 .
\end{aligned}
$$

Furthermore, we obtain the sequences $\left\{x_{n}\right\}$ and $\left\{z_{n}\right\}$ generated by iterative Algorithm 1 as

$$
\begin{aligned}
z_{n+1} & =P\left(Y_{\rho}^{\partial \psi}\left(x_{n}\right)\right)+\rho N\left(u_{n}, v_{n}, w_{n}\right) \\
& =\frac{Y_{\rho}^{\partial \psi}\left(x_{n}\right)}{2}+\frac{107 x_{n}}{420} \\
& =\frac{1}{2} \cdot \frac{3 x_{n}}{5}+\frac{107 x_{n}}{420} \\
& =\frac{3 x_{n}}{10}+\frac{107 x_{n}}{420}, \\
\text { also, } Y_{\rho}^{\partial \psi}\left(x_{n}\right) & =J_{\rho}^{\partial \psi}\left(z_{n}\right) \\
\frac{3 x_{n}}{5} & =\frac{2 z_{n}}{5} \\
x_{n} & =\frac{2 z_{n}}{5} .
\end{aligned}
$$




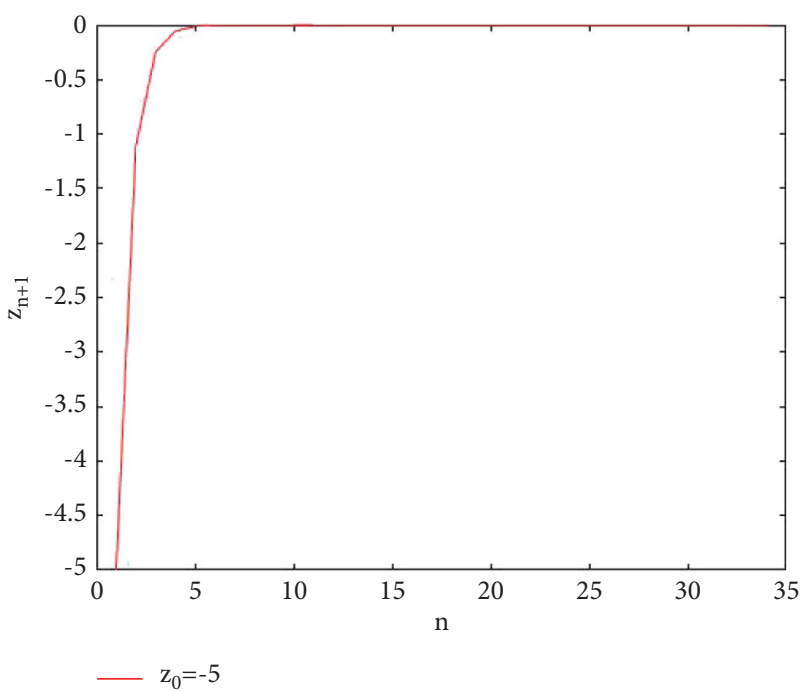

Figure 1: Case I: for the initial value $z_{0}=-5$.

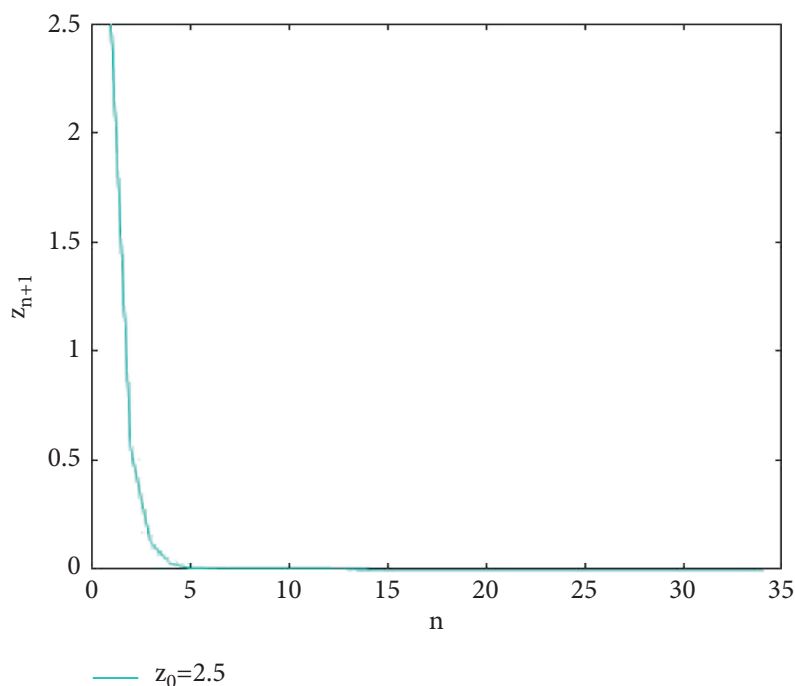

Figure 2: Case II: for the initial value $z_{0}=2.5$.

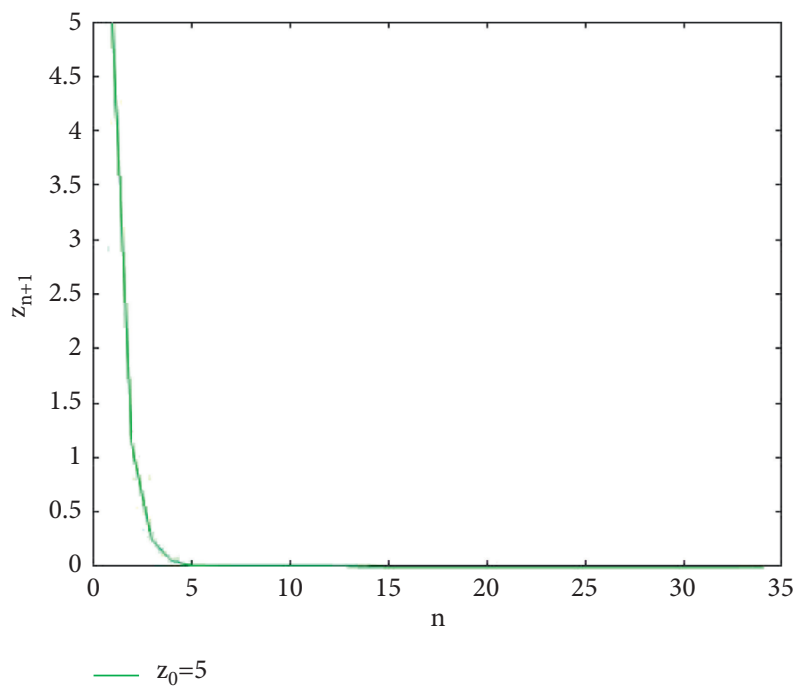

Figure 3: Case III: for the initial value $z_{0}=5$. 


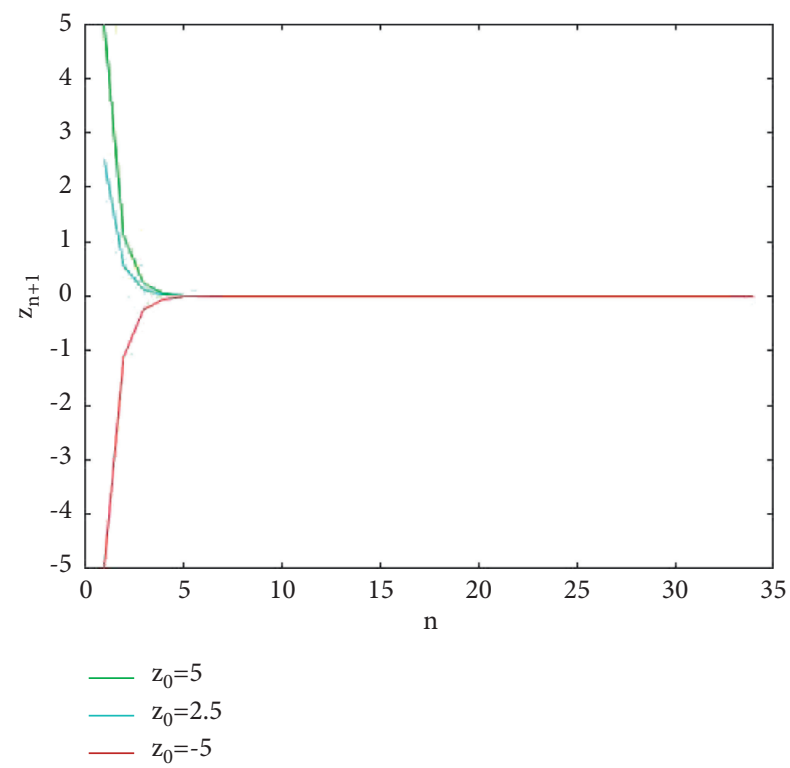

FIGURE 4: By combining all the above graphs, we get the following conjoining graph of convergence.

TABLE 1: The values of $z_{n}$ with initial values $z_{0}=-5, z_{0}=2.5$, and $z_{0}=5$.

\begin{tabular}{lccc}
\hline No. of iterations & For $z_{0}=-5$ & For $z_{0}=2.5$ & For $z_{0}=5$ \\
$z_{n}$ & 5 \\
\hline$n=1$ & $z_{n}$ & 2.5 & 1.10952380952381 \\
$n=2$ & -5 & 0.554761904761905 & 0.246208616780045 \\
$n=3$ & -1.10952380952381 & 0.123104308390023 & 0.0546348644854767 \\
$n=4$ & -0.246208616780045 & 0.0273174322427384 & 0.0121237365953486 \\
$n=5$ & -0.0546348644854767 & 0.00606186829767432 & $6.52333379900596 e-06$ \\
$n=10$ & -0.0121237365953486 & $3.26166689950298 e-06$ & $3.50996440070956 e-09$ \\
$n=15$ & $-6.52333379900596 e-06$ & $1.75498220035478 e-09$ & $1.88858189291582 e-12$ \\
$n=20$ & $-3.50996440070956 e-09$ & $9.44290946457911 e-13$ & $1.01617599469911 e-15$ \\
$n=25$ & $-1.88858189291582 e-12$ & $5.08087997349556 e-16$ & $5.00382572119910 e-17$ \\
$n=27$ & $-1.01617599469911 e-15$ & $2.50191286059955 e-17$ & $2.46397001884969 e-18$ \\
$n=29$ & $-5.00382572119910 e-17$ & $1.23198500942484 e-18$ & $5.46766680373311 e-19$ \\
$n=30$ & $-2.46397001884969 e-18$ & $2.73383340186656 e-19$ & \\
\hline
\end{tabular}

From (77) and (78), we have

$$
\begin{aligned}
& z_{n+1}=\left(\frac{3}{10}+\frac{107}{420}\right) \frac{2 z_{n}}{5}, \\
& z_{n+1}=\frac{233}{1050} z_{n} .
\end{aligned}
$$

Clearly, the sequence $\left\{z_{n}\right\}$ converges to 0 , and consequently, the sequence $\left\{x_{n}\right\}$ also converges to 0 .

It is shown in Figures 1-3 that, for initial values $z_{0}=-5,2.5$, and 5 , the sequence $\left\{z_{n}\right\}$ converges to 0 . A consolidated graph using Figures $1-3$ is provided in Figure 4. In Table 1, comparing different initial values of $\left\{z_{n}\right\}$ and for different iterations, it is obtained that the sequence $\left\{z_{n}\right\}$ converges to 0 .

\section{Conclusion}

In this work, we introduce and study three new problems, that is, a Yosida complementarity problem, a Yosida variational inequality problem, and a Yosida proximal operator equation involving XOR-operation. It is shown that Yosida complementarity problem involving XORoperation is equivalent to a Yosida variational inequality problem involving XOR-operation and Yosida variational inequality problem involving XOR-operation is equivalent to a Yosida proximal operator equation involving XORoperation. An algorithm is established to obtain the solution of Yosida proximal operator equation involving XOR-operation. Finally, an existence and convergence result is proved. A numerical example is given in support of our main result.

It is still an open and interesting problem that how to establish equivalence between Yosida complementarity problem involving XOR-operation and Yosida proximal operator equation problem involving XOR-operation.

\section{Data Availability}

No data were used to support this study. 


\section{Disclosure}

A variant form of Yosida variational inequality and Yosida proximal operator equation involving XOR-operation was considered in "Some Problems Concerning Generalized Variational Inequalities", Ph. D Thesis, (2009), AMU Aligarh [39]. In this variant form, neither the concept of Yosida approximation operator nor the concept of XOR-operation was used. Moreover, no complementarity problem was considered in the abovementioned thesis.

\section{Conflicts of Interest}

The authors declare that they have no conflicts of interest.

\section{References}

[1] G. Stampacchia, "Formes bilineaires coercitives sur les ensembles convexes," Comptes Rendus de l'Académie des Sciences, vol. 258, pp. 4413-4416, 1964.

[2] G. Ficchera, "Problemi elastostatici con vincoli unilaterali: il problema di Signorini con ambigue condizioni al contorno," Atti della Accademia Nazionale dei Lincei, Mem., Cl. Sci. Fis. Mat. Nat., Sez. Ia, vol. 7, no. 8, pp. 91-140, 1963.

[3] C. Baiocchi and A. Capelo, Variational and Quasivariational Inequalities: Applications to Free Boundary Problems, Wiley, New York, NY, USA, 1984.

[4] F. Giannessi and A. Maugeri, Variational Inequalities and Network Equilibrium Problems, Plenum Press, New York, NY, USA, 1995.

[5] R. Glowinski, J. Lions, and R. Trémolières, Numerical Analysis of Variational Inequalities, North-Holland, Amsterdam, Netherlands, 1981.

[6] D. Kinderlehrer and G. Stampacchia, An Introduction to Variational Inequalities and Their Applications, Academic Press, New York, NY, USA, 1980.

[7] J.-C. Yao, "Existence of generalized variational inequalities," Operations Research Letters, vol. 15, no. 1, pp. 35-40, 1994.

[8] R. Ahmad and Q. H. Ansari, "An iterative algorithm for generalized nonlinear variational inclusions," Applied Mathematics Letters, vol. 13, no. 5, pp. 23-26, 2000.

[9] L.-C. Ceng and M. Shang, "Generalized mann viscosity implicit rules for solving systems of variational inequalities with constraints of variational inclusions and fixed point problems," Mathematics, vol. 7, no. 10, p. 933, 2019.

[10] L.-C. Ceng, X. Qin, Y. Shehu, and J.-C. Yao, "Mildly inertial subgradient extragradient method for variational inequalities involving an asymptotically nonexpansive and finitely many nonexpansive mappings," Mathematics, vol. 7, no. 10, p. 881, 2019.

[11] K. Muangchoo, N. A. Alreshidi, and I. K. Argyros, "Approximation results for variational inequalities involving pseudomonotone bifunction in real Hilbert spaces," Symmetry, vol. 13, no. 2, p. 182, 2021.

[12] R. W. Cottle and G. B. Dantzig, "Complementary pivot theory of mathematical programming," Linear Algebra and Its Applications, vol. 1, no. 1, pp. 103-125, 1968.

[13] R. W. Cottle, Nonlinear Programs with Positively Bounded Jacobians, Ph.D Thesis, Department of Mathematics, University of California, Berkely, CA, USA, 1964.

[14] R. Petterson, "Yosida approximations for multivalued stochastic differential equations," Stochastics and Stochastics Reports, vol. 52, no. 1-2, pp. 107-120, 1995.
[15] I. Ahmad, C. T. Pang, R. Ahmad, and M. Ishtyak, "System of Yosida inclusions involving XOR-operator," Journal of Nonlinear and Convex Analysis, vol. 18, no. 5, pp. 831-845, 2017.

[16] D. V. Hieu and P. K. Quy, “An inertial modified algorithm for solving variational inequalities," RAIRO-Operations Research, vol. 54, no. 1, pp. 163-178, 2020.

[17] M. Ertürk, F. Gürsoy, and N. Şimşek, "S-iterative algorithm for solving variational inequalities," International Journal of Computer Mathematics, vol. 98, no. 3, pp. 435-448, 2021.

[18] L.-C. Ceng and Q. Yuan, "Systems of variational inequalities with nonlinear operators," Mathematics, vol. 7, no. 4, p. 338, 2019.

[19] Z. A. Rather, R. Ahmad, and C.-F. Wen, "Variational-like inequality problem involving generalized Cayley operator," Axioms, vol. 10, no. 3, p. 133, 2021.

[20] Y. J. Cho, J. Li, and N.-j. Huang, "Solvability of implicit complementarity problems," Mathematical and Computer Modelling, vol. 45, no. 7-8, pp. 1001-1009, 2007.

[21] J.-C. Yao, "On the generalized complementarity problem," The Journal of the Australian Mathematical Society. Series B. Applied Mathematics, vol. 35, no. 4, pp. 420-428, 1994.

[22] S. Schaible and J.-C. Yao, "On the equivalence of nonlinear complementarity problems and least-element problems," Mathematical Programming, vol. 70, no. 1-3, pp. 191-200, 1995.

[23] J.-C. Yao, "A basic theorem of complementarity for the generalized variational-like inequality problem," Journal of Mathematical Analysis and Applications, vol. 158, no. 1, pp. 124-138, 1991.

[24] Y. I. Alber, "Proximal projection methods for variational inequalities and Cesáro averaged approximations," Computers \& Mathematics with Applications, vol. 43, no. 8-9, pp. 1107-1124, 2002.

[25] M. T. Hoang and O. F. Egbelowo, "Nonstandard finite difference schemes for solving an SIS epidemic model with standard incidence," Rendiconti del Circolo Matematico di Palermo Series 2, vol. 69, no. 3, pp. 753-769, 2020.

[26] C. C. Okeke and C. Izuchukwu, "Strong convergence theorem for split feasibility problems and variational inclusion problems in real Banach spaces," Rendiconti del Circolo Matematico di Palermo Series 2, vol. 70, no. 1, pp. 457-480, 2021.

[27] M. Rahaman, R. Ahmad, M. Dilshad, and I. Ahmad, "Relaxed $\mathrm{H}$-proximal operator for solving a variational-like inclusion problem," Mathematical Modelling and Analysis, vol. 20, no. 6, pp. 819-835, 2015.

[28] R. Ahmad and A. H. Siddiqi, "Mixed variational-like inclusions and J $\eta$-proximal operator equations in Banach spaces," Journal of Mathematical Analysis and Applications, vol. 327, no. 1, pp. 515-524, 2007.

[29] H. G. Li, "Nonlinear inclusion problems for ordered RME Setvalued mappings in ordered Hilbert spaces," Nonlinear Functional Analysis and Applications, vol. 16, pp. 1-8, 2001.

[30] X. P. Ding and F. Q. Xia, "A new class of completely generalized quasi-variational inclusions in Banach spaces," Journal of Computational and Applied Mathematics, vol. 147, no. 2, pp. 369-383, 2002.

[31] Q. H. Ansari, C. S. Lalitha, and M. Mehta, Generalized Convexity, Nonsmooth Variational Inequalities, and Nonsmooth Optimization, Taylor \& Francis, Abingdon, UK, 2014.

[32] N.-J. Huang, J. Li, and D. O'Regan, "Generalized -complementarity problems in Banach spaces," Nonlinear Analysis: Theory, Methods \& Applications, vol. 68, no. 12, pp. 38283840, 2008. 
[33] H. Yin and C. Xu, "Vector variational inequality and implicit vector complementarity problems," Vector Variational Inequalities and Vector Equilibria, vol. 38, pp. 491-505, 2000.

[34] F. Flores-Bazán and R. López, "The linear complementarity problem under asymptotic analysis," Mathematics of Operations Research, vol. 30, no. 1, pp. 73-90, 2005.

[35] G. Isac, "Complementarity problems," in Lecture Notes in Mathematics, vol. 1528, Berlin, Germany, Springer-Verlag, 1992.

[36] G. Isac, "On the implicit complementarity problem in Hilbert spaces," Bulletin of the Australian Mathematical Society, vol. 32, no. 2, pp. 251-260, 1985.

[37] A. Farajzadeh and A. A. Harandi, "Generalized complementarity problems in Banach spaces," Albanian Journal of Mathematics, vol. 3, pp. 35-42, 2009.

[38] S. Nadler, "Multi-valued contraction mappings," Pacific Journal of Mathematics, vol. 30, no. 2, pp. 475-488, 1969.

[39] F. Usman, Some Problems Concerning Generalized Variational Inequalities, Ph.D Thesis, AMU Aligarh, Aligarh, India, 2009, http://ir.amu.ac.in/3124/1/T\%207060.pdf. 